\title{
LA RESECCIÓN TRANSURETRAL CON SOLUCIÓN SAUNA: UN LOGRO TECNOLÓGICO AÚN NO ASIMILADO POR LA UROLOGÍA.
}

José G abriel Valdivia Uría, O scar Regojo Zapata, José M anuel Sánchez Zalabardo, Angel Elizalde Benito, Joaquín N avarro G il y Ignacio Hijazo Conejos.

Servicio de Urología del Hospital Clínico Universitario "Lozano Blesa". Zaragoza. España

\begin{abstract}
Resumen.- O BJETIVO: Demostrar objetivamente las ventajas que reporta el uso de la R.T.U.P. con solución salina (logro posible gracias al desarrollo tecnológico de los los nuevos generadores de corrientes de alta frecuencia), pero que curiosamente ha sido recibida por los urólogos con cierto escepticismo.

MÉTO DO S: A un primer grupo de 51 pacientes (grupo A) se les sometió a R.T.U.P., a baja presión hidráulica, con un sistema bipolar por pulsos (G yrus Plasmakinetic, con asa large loop) y empleando suero fisiológico como líquido irrigante. A un segundo grupo, de 49 pacientes se les practicó una R.T.U.P., a baja presión hidráulica, con un sistema monopolar convencional (Erbe 350 ) y empleando solución de glicina con etanol como líquido irrigante.
\end{abstract}

RESULTADO S: En el primer grupo de pacientes se obtuvo un mejor rendimiento quirúrgico y menor grado de hemorragia. No se produjeron complicaciones intraoperatorias en ambos grupos. Solo se registró un caso de hemorragia tardía al mes de la intervención en un paciente del grupo $A$.

CO N C LUSIO N ES: La R.T.U. bipolar por pulsos, tiene una mínima inercia térmica en profundidad, es capaz de hemostasiar los vasos por desecación en vez de carbonización, dispone de una calidad de corte extremadamente preciso, no provoca estímulo neuromuscular y hace posible el uso de una solución salina como medio de irrigación. Todo ello se traduce en un menor daño tisular, nulo riesgo de secuelas por fugas de corriente, una técnica más depurada, un menor riesgo de perforaciones accidentales, y la posibilidad de duplicar o triplicar sin riesgo el tiempo quirúrgico, especialmente si se trabaja con baja presión hidráulica.

Palabras clave: Resección transuretral.

Resectoscopio. Electrobisturí. Bisturí bipolar. R.T.U.P. bipolar.

Summary.- O BJEC TIVES: To objectively demonstrate the advantages provided by TURP with saline solution (an adjustment made possible thanks to the technological development of new high frequency current genera tors), although it surprisingly has been received with scepticism in the urological community.
José G abriel Valdivia Uría.

Servicio de Urología.

H. Clínico Universitario Lozano Blesa.

Avenida San Juan Bosco, 15.

50009 Zaragoza. (España)

e-mail: uroh@hcu-lblesa.es

Trabajo recibido: 29 de octubre 2004 
METHODS: A first group of 51 patients (group A) underwent low hydraulic pressure TURP with a pulsed bipolar system (G yrus Plasmakinetic, with large loop) using physiologic saline solution as irrigation. A second group of 49 patients underwent low hydraulic pressure TURP with a conventional monopolar system (Erbe 350 ) using glycine-ethanol solution.

RESULTS: A better surgical performance was obtained in the first group, as well as a lower degree of bleeding. No intraoperative complications appear in either group. O nly one case of late hematuria was registered one month after surgery in a patient of group $A$.

CONCLUSION S: Pulsed bipolar TURP has a minimal thermal in-depth diffusion, achieves vessel hemostasis by dessication instead of charring, has an extremely precise cutting quality, does not produce neuromuscular stimulation, and makes the use of saline solution irrigation possible. All these translate to lower tissue injury, null risk of sequelae secondary to electric current leak, a finer technique, a lower risk of accidental perforation, and the possibility of duplicate or triplicate the surgical time without risk, especially when working with low hydraulic pressure.

Keywords: Transurethral resection. Resectoscope. Electric scalpel. Bipolar energy. Bipolar TURP.

\section{INTRODUCCIÓN}

En la última década el mercado tecnológico ha tratado de introducir, mas bien con poca fortuna, diversas alternativas a la resección transuretral de la próstata (R.T.U.P.), que sigue siendo el patrón de oro del tratamiento quirúrgico de hiperplasia benigna de la próstata.

Cabe atribuir a la postura generalizada de rechazo ante tantas innovaciones frustradas, el que la inmensa mayoría de los urólogos no haya aceptado aún el hecho de que con la resección bipolar por pulsos se han mejorado muy ostensiblemente las grandes ventajas que siguen consolidando a la R.T.U. como la técnica mínimamente invasiva por excelencia.

Con el advenimiento de la videocirugía, la R.T.U. pasó a ser del dominio de todos, y son muchos los urólogos que consideran ya como simple referencia histórica el límite máximo de los 50 gramos en el planteamiento de su indicación. No obstante, siguen produciéndose, en un número no desdeñable de casos, algunas complicaciones como la hemorragia 0 el síndrome de la resección transuretral (1), y secuelas como esclerosis de celda, estenosis uretral, incontinencia, impotencia, etc. que empañan los brillantes resultados de esta técnica (2).

La R.T.U. bipolar por pulsos, al tener sólo una mínima inercia térmica en profundidad, hemostasiar los vasos por desecación en vez de carbonización, disponer de una calidad de corte extremadamente preciso, no provocar electroestimulación neuromuscular y permitir la utilización de una solución salina como medio de irrigación, ofrece al urólogo innegables ventajas, a las que cabe añadir también las aplicaciones que tienen este tipo de corrientes en cirugía abierta y laparoscópica (especialmente el sellado de vasos de hasta $7 \mathrm{~mm}$ de diámetro) (3).

\section{CONCEPTOS BÁSICOS DE ELECTROCIRUGÍA}

El urólogo es uno de los especialistas que con más frecuencia utiliza instrumentos electroquirúrgicos, y por tanto tiene la responsabilidad de conocer los principios básicos de su funcionamiento. De lo contrario se expone a provocar al paciente, a si mismo o a otros miembros del equipo quirúrgico, lesiones que pueden dejar secuelas irreversibles o incluso causar la muerte.

Para comprender el funcionamiento de los nuevos bisturíes bipolares por pulsos, es preciso recordar algunos conceptos básicos sobre electrocirugía.

La electricidad consiste en una corriente de electrones capaz de fluir libremente a través de substancias conductoras y no en cambio a través de otras llamadas aislantes.

El voltaje es la fuerza que se necesita para empujar a esos electrones a través de un alambre, tal como la que haría una bomba para impulsar agua por una tubería.

Los amperios miden en cambio la tasa de flujo de electrones a través del alambre, como si fuera el volu- 
men de agua que corre por esa tubería. La electricidad fluye pues por un alambre como el agua lo hace dentro de una tubería, con una determinada fuerza y un caudal que pueden utilizarse para llevar a cabo un determinado trabajo.

La potencia de la corriente eléctrica es el producto de la fuerza (voltaje) y el flujo (amperios) y se mide en watios.

Circuito electroquirúrgico: A diferencia del agua que fluye por una tubería y escapa al final de la misma, los electrones para poder fluir deben de completar un circuito cerrado. En el caso de los electrobisturíes monopolares, este circuito se cierra cuando la corriente fluye desde el generador hacia el electrodo activo para pasar a través del paciente y regresar a través del electrodo indiferente. Sin embargo, en los electrobisturíes bipolares, la corriente fluye alternativamente del generador a través de uno de los electrodos, atraviesa el tejido interpuesto entre éste y el otro electrodo, y retorna al generador por este último.

La resistencia o impedancia que oponen los tejidos al paso de electrones se mide en ohmios, y desde el punto de vista conceptual es muy importante saber que es la resistencia que encuentran los electrones a su paso por un conductor lo que genera calor, y que en electrocirugía es la resistencia tisular y no el electrodo activo quien genera el calor.

La densidad de la potencia del instrumento electroquirúrgico es otro factor que debe conocer muy bien el urólogo. La densidad de potencia es la cantidad de potencia entregada, dividida por el área sobre la cual se aplica ésta. Así, cuando con un electrobisturí monopolar se aplica energía mediante un electrodo activo en un área muy pequeña (el asa del resectoscopio, por ejemplo), la alta densidad de potencia provoca una vaporización o coagulación controladas. Sin embargo, como la energía que sale del paciente lo hace a través de un electrodo muy amplio (la placa neutra del electrobisturí) no se producen lesiones térmicas en su zona de contacto, (4).

En la corriente continua, el flujo de electrones a través del circuito eléctrico se realiza en un solo sentido y sin ningún tipo de fluctuación. Este tipo de corriente se usa aún ocasionalmente en microcirugía experimental (electrocauterio).
La corriente alterna supone un ir y venir de los electrones en uno y otro sentido del circuito eléctrico. Según la frecuencia con que se produce este cambio de ciclo (ondas) se distinguen corrientes de baja, media y alta frecuencia.

Características de la ondas: La altura o amplitud del pico de una onda representa el voltaje, la longitud de onda es la distancia que existe entre dos picos y la frecuencia es el número de ondas que se generan en un segundo.

Esta frecuencia se mide en Herzios (un kHz son 1000 ondas por segundo). La mayoría de los electrobisturíes trabaja con frecuencias entre 400 y $1000 \mathrm{kHz}$. Las frecuencias por debajo de $100 \mathrm{kHz}$ no pueden ser utilizadas ya que generan un fuerte estímulo en el sistema neuromuscular. Estas bajas frecuencias son las responsables de la típica contractura de los músculos adductores, por estímulo del nervio obturador en el curso de las R.T.U. cuando la vejiga está distendida y se aproxima mucho el asa de corte a este nervio y nuestro electrobisturí genera algunas corrientes artefactadas de baja frecuencia. También en la R.T.U.P. suelen observarse contracciones involuntarias del músculo elevador del ano durante la exéresis del ápex prostático.

Tipos de ondas: simplificando los términos, podemos decir que los electrobisturíes pueden generar corrientes con tres tipos de ondas: "ondas puras" caracterizadas por la uniformidad de sus oscilaciones, "ondas amortiguadas" caracterizadas por el frenado que presentan sus oscilaciones a poco de ser generadas, y "ondas mixtas" que son una mezcla de las dos anteriores.

- Las "ondas puras" generan un calor muy intenso, capaz de hacer estallar a las células por ebullición, vaporizándolas en el acto, lo cual las hace muy apropiadas para el corte tisular, utilizando un electrodo muy fino con el fin de obtener una alta densidad de corriente en el área de corte.

- Las "ondas amortiguadas", a unque inicialmente tienen picos de voltaje más elevados, éstos se frenan enseguida, lo que hace posible un relativo enfriamiento tisular entre un ciclo y otro. Por ello, el tejido se deseca, coagula o carboniza, pero no se vaporiza.

- Las "ondas mixtas" fueron diseñadas para cortar y al mismo tiempo coagular los tejidos. No son 
apropiadas para la R.T.U. ya que aunque evitan en cierto modo la intensidad de la hemorragia, tienen una elevada inercia térmica que ocasiona una profunda mortificación tisular de la celda, que retrasa la cicatrización y provoca una molesta irritabilidad miccional en el postoperatorio.

\section{ELECTROBISTURÍES EMPLEADOS EN RESEC- CION TRANSURETRAL}

Los electrobisturíes monopolares utilizados en las R.T.U. generan corrientes de alta frecuencia aptas para realizar corte puro o coagulación, si bien son capaces también de entregar corrientes mixtas. Lo ideal es que estos electrobisturíes generen corrientes muy puras, de alto voltaje (entre 300 y 900 voltios), alta frecuencia (entre $300 \mathrm{KHz}$ y $2 \mathrm{M} \mathrm{Hz}$ ) y bajo amperaje (250 mA), capaces de atravesar el cuerpo humano sin producir otros daños que los estrictamente calculados en la zona de contacto del electrodo activo.

Los electrobisturíes bipolares convencionales, utilizados hasta ahora en cirugía abierta o laparoscópica, tienen una capacidad de potencia de salida muy inferior a la de los electrobistuíes monopolares. Las terminales (pinzas) con las que se aplica este tipo de corriente precisan cerrar el circuito apresando el tejido entre sus ramas, con lo cual se produce un efecto loca-

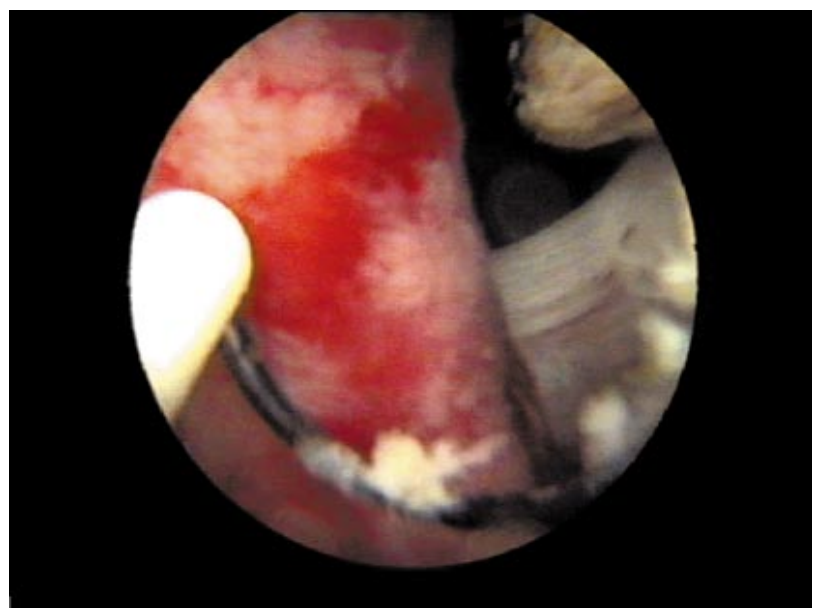

FIG URA 1. Primeros momentos de la R.T.U.P. descubriéndose fibras de cuello en horario 5 oㅡ. lizado y muy limitado de desecación y coagulación. O bviamente, no son aptos para la electrorresección transuretral, ya que además precisan que el medio en el que se aplican no se encuentre sumergido en sangre o soluciones electrolíticas. Tienen en cambio la ventaja de que la corriente eléctrica no atraviesa el cuerpo del paciente en busca del electrodo de salida (la placa neutra), hecho que suele ser causa de múltiples problemas: calentamiento en profundidad, con desecación y necrosis del tejido próximo al área de trabajo, posibles interferencias con marcapasos, riesgo de quemaduras, electrocución, etc.

Los electrobisturíes que generan corrientes de alta frecuencia por pulsos son equipos de nuevo diseño, que utiliza el lla mado "sistema de tratamiento tisular plasmakinético", desarrollado inicialmente por la firma GYRUS Medical Ltd. Bucks, UK, y que ahora fabrican también otras empresas, empleando diferentes nomenclaturas que no hacen otra cosa que crear confusión en los no avezados.

Estos generadores producen altos niveles de energía, en ciclos instantáneos de pulsos (que provocan un rápido incremento de temperatura en los tejidos), intercalados por cortos periodos de pausa, con lo que se logran unos efectos de coagulación tisular muy uniforme y completa, capaces de sellar vasos de hasta $7 \mathrm{~mm}$ cuando esta energía se transmite a través

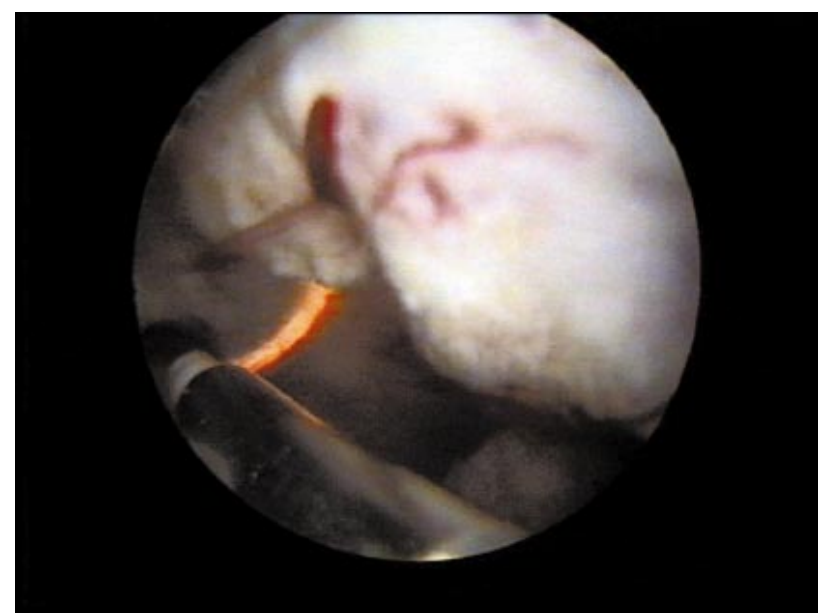

FIG URA 2. Aspecto que ofrece la corona de plasma durante el corte del adenoma. Este plasma se genera gracias a la presencia de los electrolitos del suero fisiológico. 
de unas pinzas de prensión apropiadas. Estas cualidades han hecho que el uso de estos equipos se haya generalizado más en cirugía abierta o laparoscópica que en cirugía endourológica. Resulta curioso observar cómo algunos urólogos los utiliza en estas otras aplicaciones con el convencimiento de que se trata de instrumentos ultrasónicos...

En la R.T.U. lo que se aprovecha de estos equipos es la capacidad que tiene su corriente de corte de crear un arco de plasma alrededor del asa del resectoscopio. Con un ajuste adecuado de los microsegundos de los pulsos y de la energía liberada, y manteniendo al asa rodeada por solución salina se consigue crear esta corona de plasma ionizado. El tejido alcanzado por la intensa energía cinética de la corona se reduce al instante a sus elementos constitutivos (se volatiliza). Pero la ventaja fundamental reside en la baja inercia térmica del plasma, que evita daños en el tejido adyacente a la zona de aplicación. Esto, y el hecho de tratarse de una corriente bipolar, evitan la desecación del tejido más profundo, con lo que no se pierde eficacia y puede seguir cortándose éste con suma facilidad.

El empleo de términos como "volatilizar" o "vaporizar" puede inducirnos a pensar erróneamente que estamos una vez más frente a una variante de los "rollerballs" o "rollerbars" para electrovaporización prostática. A unque los electrobisturíes bipolares por pulsos pueden ser utilizados (muy ventajosamente) para

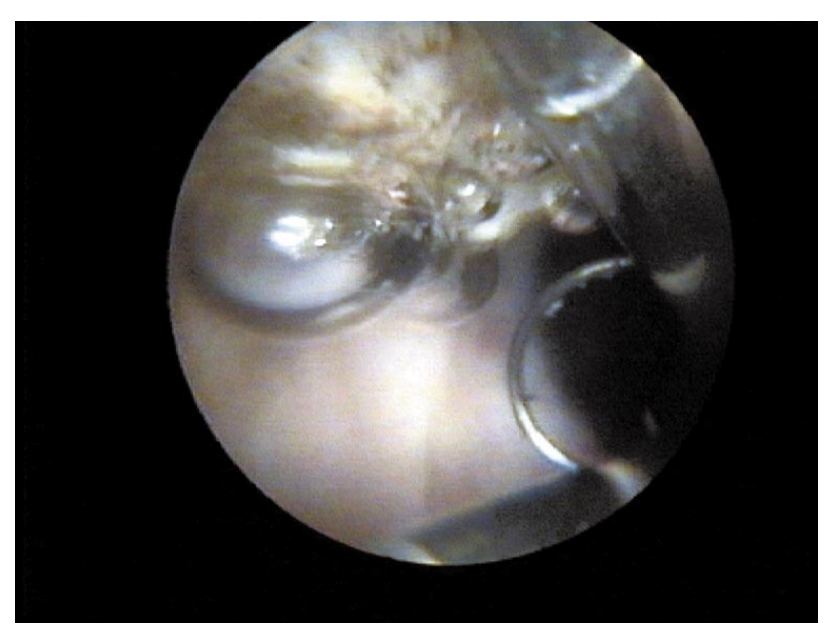

FIG URA 3. El medio de trabajo es muy claro, dada la escasa hemorragia que se produce durante la resección. estos fines, aquí de lo que estamos tratando es de la técnica de la resección transuretral clásica, llevada a cabo con asa de corte, pero utilizando un generador de corrientes de alta frecuencia, bipolar, por pulsos, y trabajando con suero fisiológico como líquido irrigante.

\section{RESECTOSCOPIOS Y NUEVAS ASAS}

Una gran ventaja de esta nueva forma de energía electroquirúrgica es que no requiere la renovación de todo el armamentario de resección transuretral. No obstante, algo que llama la atención al neófito es que en algunos de estos equipos el asa del resectoscopio es simple y en otros es doble.

La casa GYRUS en su equipo PK Plasma-Sect Large Loop ${ }^{\text {TM }}$ y la casa 0 lympus en su equipo UES-40 SurgM aster TURISTM emplean un asa simple, pero dado que las varillas del asa deben conducir la corriente eléctrica en uno y otro sentido, es decir, del y hacia el generador, el elemento de trabajo en el que se engastan los vástagos del asa tiene que ser algo diferente. Como es lógico, aunque aparentemente el asa tiene un aspecto similar al de las convencionales, su estructura es diferente. El recubrimiento externo de los extremos de las varillas actúa como un polo y el alambre del asa como otro. Entre ambos se intercalan unas cortas barritas de cerámica aislante.

La casa ACMI ha diseñado para su resectoscopio bipolar VISTA ${ }^{T M}$ un asa doble (dispuestas una

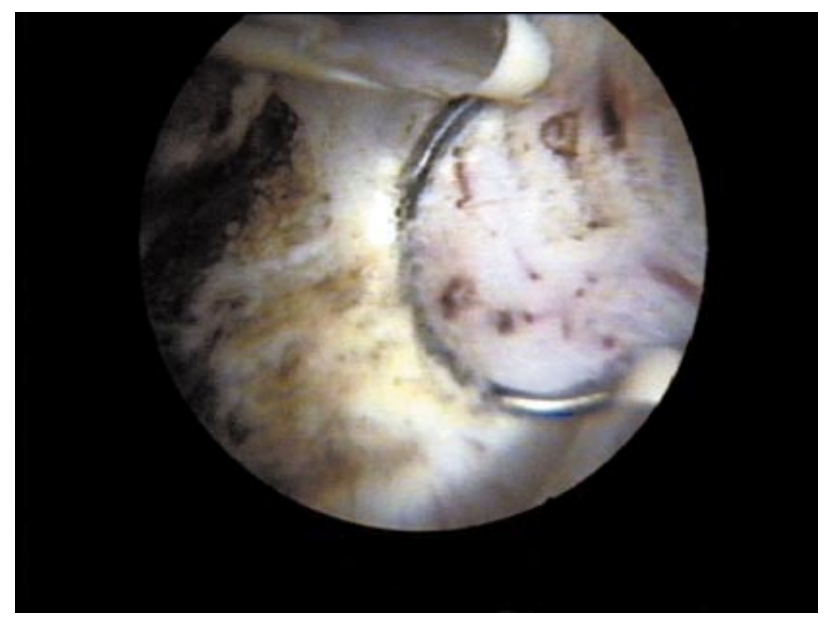

FIG URA 4. Hemostasia por desecación, sin carbonización. Las manchas oscuras se deben a la presencia de litiasis prostática 
delante de otra y separadas entre si por un par de $\mathrm{mm}$ ), de modo que su proximidad facilita la formación del arco de plasma.

\section{MATERIAL}

\section{Equipo utilizado:}

El electrobisturí bipolar por pulsos utilizado por nosotros es de la marca $G$ yrus ${ }^{\mathrm{TM}}$ y consta de los siguientes elementos:

A) Generador Plasmakinético: este equipo genera una potencia mucho más elevada que un electrobisturí bipolar convencional ( $200 \mathrm{~W}, 160 \mathrm{ohms}$ ) puesto que ha de permitir trabajar en un medio líquido de muy alta conductibilidad. Su rango de radiofrecuencia oscila entre 320 y $450 \mathrm{kHz}$ y su rango de voltaje entre 254 y $350 \mathrm{~V}$.

El generador reconoce automáticamente el código interno del asa del resectoscopio y configura por defecto unos valores, que en este caso son PK3 160 (corte) y DES 80 (hemostasia). Estos valores pueden ser modificados, a juicio del urólogo, si así lo estima necesario. El valor PK3 podría modificarse desde 5 hasta 200 y el DES desde 5 a 120.

B) Conmutador: muy similar al de cualquier electrobisturí, el pedal izquierdo (de color azul) selecciona corriente de corte mediante vaporización, en

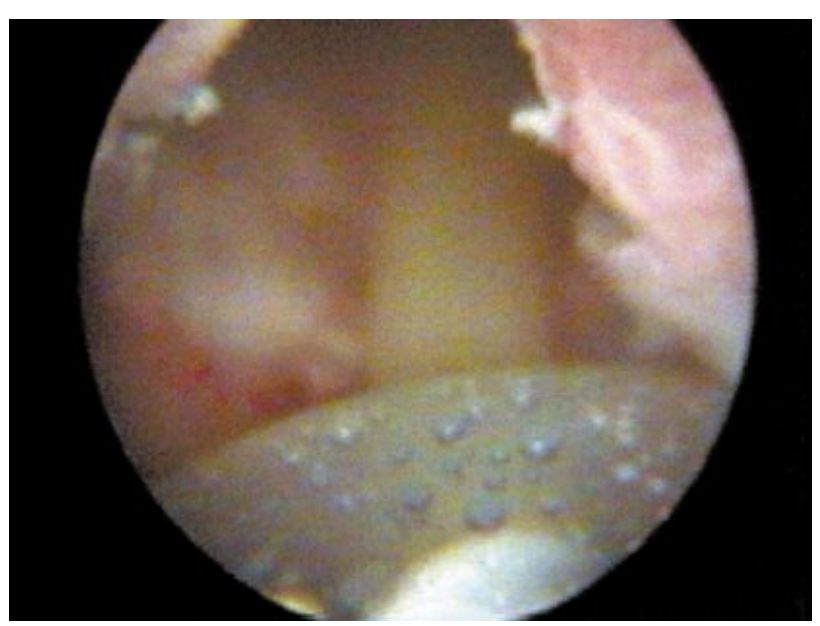

FIG URA 5. Imagen obtenida desde el Amplatz suprapúbico, de la celda prostática y el globo de la sonda al final de la R.T.U.P. O bsérvese la claridad del medio. tanto que el pedal derecho (amarillo) sirve para seleccionar corriente de desecación tisular, con efecto de hemostático.

C) Cable de conexión: este cable, de tres metros de longitud, conecta la salida del generador (situada en su panel frontal) con el cable estéril adosado al asa del resectoscopio.

D) Resectoscopios: hemos utilizado indistintamente resectoscopios convencionales Storz, de $27 \mathrm{Fr}$, provistos de un elemento de trabajo activo (Gyrus) y un resectoscopio de irrigación succión continua $G$ yrus, de $27 \mathrm{Fr}$, , provisto también de un elemento de trabajo (en este caso, pasivo). A mbos elementos de trabajo están ligeramente modificados para que puedan alojar el mecanismo de conexión y fijación del asa, que difiere del de un asa convencional. Existen también elementos de trabajo (Gyrus) que pueden ser utilizados con resectoscopios Wolf.

E) A sas de corte: por el momento, sólo existen en el mercado dos tipos de asas de corte Plasmakinético. Una de tan solo $3 \mathrm{~mm}$ de diámetro (PK Plasma - Sect ${ }^{\mathrm{T}}$ ), que fue aprobada por la FDA el 11 de Mayo de 2000 para ser usada en resecciones transuretrales de próstata y tumores de vejiga (hoy ya en desuso) y otra de más reciente aparición, con diámetro de $4 \mathrm{~mm}$ (PK Plasma - Sect Large Loop ${ }^{\mathrm{TM}}$ ), que permite obtener un mayor rendimiento en la resección y que es la que hemos utilizado en esta serie.

El asa va provista de un clip de estabilización que abraza parcialmente al eje de la óptica, de un pequeño cono de goma (que hace las veces de tapón y de cojinete a la varilla del asa) y de un cable eléctrico fijo a su extremo proximal mediante un codo reforzado que hace las veces de enganche al elemento de trabajo.

\section{Pacientes intervenidos}

Con el fin de valorar con objetividad las ventajas e inconvenientes que reporta el empleo del equipo Plasmakinético, hemos realizado un estudio retrospectivo sobre 100 R.T.U.P. realizadas en el Servicio de Urología del Hospital Clínico Universitario de Zaragoza en los últimos 24 meses. A 51 pacientes de esta serie se les realizó la R.T.U.P con el sistema Plasmakinético Grus $^{\mathrm{TM}}$ (grupo $\mathrm{A}$ ) y a 49 utilizando un electrobisturí monopolar Erbe ICC $350^{\mathrm{Tm}}$ (grupo B). Como líquido de irrigación en los pacientes del primer grupo se utilizó suero fisiológico y en los del segundo, solución de glicina al 1,5\% con etanol al $1 \%$. 
Los criterios de inclusión de los pacientes en ambos grupos fueron los que habitualmente, y con elasticidad, se utilizan en nuestro Servicio, admitiéndose para R.T.U.P. adenomas prostáticos con volúmenes de hasta $100 \mathrm{ml}$ en la ecografía abdominal. No obstante, el volumen de las próstatas de los pacientes incluidos en este estudio osciló entre 14 y $120 \mathrm{ml}$. No se realizaron ni aleatorización ni selección de los casos, sino que la incorporación de pacientes a uno u otro grupo dependió exclusivamente de la disponibilidad de uso del equipo y de las preferencias de los cirujanos reseccionistas. Estas intervenciones fueron realizadas por 7 diferentes urólogos de staff y 3 residentes del Servicio de Urología.

La edad media de los pacientes del grupo A era de 70,4 años y la del grupo B de 70,7. Todos ellos tenían un riesgo anestésico entre ASA II y ASA III

\section{MÉTODO}

Salvo a 5 pacientes ( 3 del grupo A y 2 del B) que por diversas circunstancias se les realizó una anestesia general, a todos los demás se les realizó una a nestesia intradural.

La técnica de la R.T.U.P la practicamos siguiendo el concepto de baja presión hidráulica real. Ello supone que las bolsas de líquido irrigante están situadas a tan solo $40 \mathrm{~cm}$ de altura sobre la sínfisis púbica, y que la vejiga se drena intraoperatoriamente mediante simple sifonaje a través de una camisa de A mplat de $30 \mathrm{fr}$. conectada a un tubo de polivinilo de $90 \mathrm{~cm}$. Esta sistemática fue seguida también en los casos operados con bisturí bipolar por pulsos.

Lo más significativo es que en estos casos hay que utilizar suero fisiológico como líquido de irrigación (en ningún caso puede funcionar con agua destilada o una solución de glicina) y que no es necesario colocar al paciente una placa indiferente de electrobisturí.

Como es rutinario en nuestro Servicio, en los pacientes a los que se les practica R.T.U.P. con solución de glicina (grupo B) se monitoriza la posible reabsorción de líquido irrigante durante la intervención e inmediatamente después de la misma con el test de etanol en aire espirado, (tan solo a un paciente del grupo A pudo realizarse este tipo de monitorización dadas las dificultades encontradas hasta ahora para conseguir solución fisiológica con etanol al $1 \%$ ). Por motivos técnicos, la monitorización con etanol tampoco pudo realizarse a los pacientes sometidos anestesia general.

Como en cualquier R.T.U.P. la intervención se inicia con una inspección vesicouretral y el cálculo mental de la longitud de la uretra prostática.

En la R.T.U.P. bipolar, el asa se activa unos instantes antes de apoyarse en el tejido adenomatoso. En cuando se ha iniciado el corte, se observa la aparición de una corona de fuego (plasma) rodeando al asa. Para su activación, el generador intensifica automáticamente la potencia de salida en un $25 \%$ durante los primeros 400 milisegundos del corte. El movimiento imprimido al asa es el habitual pero procurando dar siempre cortes profundos, largos y uniformes. La estrategia en cuanto a abordaje del adenoma depende de la costumbre de cada urólogo, así como de la morfología del propio adenoma.

En nuestro caso, la resección solemos iniciarla siempre en horario 5o (salvo que exista un lóbulo medio muy prominente), realizando profundos y largos cortes desde la vertiente vesical del cuello hasta las proximidades del veru montanum. Con estos cortes se profundiza hasta descubrir las fibras de cuello vesical y la trama típica de "tejido de mimbre" de la cápsula prostática. Se alargan entonces los cortes hasta el límite con la uretra membranosa. Curiosamente, con este tipo de bisturíes, el tejido capsular suele aparecer mucho más sonrosado que el adenomatoso, lo cual facilita mucho la identificación de estas estructuras.

Los siguientes cortes se dan en horario 600 y luego en horario 70o, siguiendo los mismos pasos que en horario 500 . Una vez revisada la uniformidad de la cápsula y completada la hemostasia, se reseca, de abajo arriba la porción alta de un lóbulo lateral, hasta alcanzar el horario 12ㅇ․ Revisada la uniformidad de la cápsula a este nivel y completada la hemostasia, se realiza lo mismo en la porción lateral y alta del lóbulo contralateral.

Antes de concluir la intervención, se revisa nuevamente la uniformidad de la cápsula se repasan los contornos de cuello vesical y de uretra prostática y se completa la hemostasia.

Concluida la intervención, y una vez extraídos los fragmentos resecados, en los casos en los que uti- 
lizamos el resectoscopio de irrigación succión continua (G yrus) colocamos al paciente una sonda de tres vías, de $20 \mathrm{Fr}$. En los casos en los que utilizamos un resectoscopio convencional Storz, con camisa de Amplatz suprapúbica (tal como es nuestra sistemática habitual de trabajo), dejamos una sonda Folatex hematuria de $20 \mathrm{Fr}$ (de 2 vías) por vía uretral y una sonda Foley de $14 \mathrm{Fr}$ suprapúbica.

Los fragmentos de resección se pesan sistemáticamente en un pesacartas mecánico y se anota este dato así como cualquier otro relevante en el protocolo quirúrgico de la intervención.

Toda la información referente a los pacientes y a las incidencias quirúrgicas se introduce en una base de datos en Stat view T.M. para su posterior análisis estadístico. Utilizamos el text Anova para la comparación de medias de las variables cuantitativas y el text de Chi cuadrado para las variables cualitativas.

\section{RESULTADOS}

La técnica quirúrgica se llevó a cabo en todos los pacientes sin ningún contratiempo, a pesar de que en los pacientes del grupo A se produjeron más perforaciones capsulares $(37,2 \%)$ que en el grupo B $(22,4 \%)$. El peso medio de tejido adenomaoso obtenido en los pacientes del grupo A fue de 47,24 gr., en tanto que en los del grupo B fue de $46,22 \mathrm{gr}$.
La duración media de la R.T.U.P. fue de 55,2 minutos en los pacientes del grupo $A$ y de 62,8 minutos en los del B. De acuerdo con estos datos podemos deducir que el rendimiento de la resección en los pacientes del grupo A fue de 0,92 gramos / minuto, en tanto que en los del grupo $B$, de 0,70 gramos / minuto. Se encontró que la diferencia de medias era estadísticamente significativa para las variables de tiempo de intervención y velocidad de resección (Tabla I).

Solo fue preciso trasfundir intra o peratoriamente a tres pacientes del grupo A (todos ellos con hematocrito bajo antes de la intervención) en tanto que 6 pacientes del grupo B precisaron hemotransfusión (sólo 2 de éstos tenía hematocrito bajo antes de la intervención).

Todos los pacientes operados con el sistema Plasmakinético $G$ yrus $^{\mathrm{TM}}$ evolucionaron de manera muy satisfactoria. No obstante, hemos registrado un caso de reingreso, al mes de la intervención, por hematuria intensa que llegó a provocar vejiga coagulada, entre los pacientes del grupo A. Este paciente precisó de reposición sanguínea y de una electrocoagulación puntual del vaso sangrante.

La estancia media hospitalaria de los pacientes del subgrupo A fue de 5,6 días, en tanto que en los del $B$ fue de 7,4 siendo la diferencia estadísticamente significativa $(p=0,001)$.

TABLA I. COM PARACIÓN DE MEDIAS DE LAS DIFEREN TES VARIABLES EN LO S DOS G RUPOS.

\begin{tabular}{|l|c|c|c|c|}
\hline & R.T.U.P. bipolar por pulsos & R.T.U.P. monopolar & total & $\mathrm{p}$ \\
\hline $\mathrm{n}$ - pacientes & 51 & 49 & 100 & \\
\hline edad media & 70,4 & 70,7 & & 0,84 \\
\hline tiempo operatorio (min) & 55,2 & 62,8 & & 0,008 \\
\hline peso tej resecado (gr) & 47,24 & 46,22 & & 0,83 \\
\hline velocidad de resección (g/ min) & 0,92 & 0,70 & & 0,0005 \\
\hline estancia media & $5,6 \mathrm{~d}$ & $7,4 \mathrm{~d}$ & & 0,001 \\
\hline \% transfusión & 5,8 & 12,2 & 9 & 0,266 \\
\hline perforación capsular (\%) & 37,2 & 22,4 & & 0,08 \\
\hline
\end{tabular}


En ninguno de los pacientes de ambos subgrupos hemos registrado cuadros de irritabilidad vesical, como se han descrito cuando se emplean técnicas de foto o electro vaporización prostática, de ablación prostática por fotocoagulación, alcoholización, radiofrecuencia, ultrasonidos focalizados, termoterapia, piroterapia, etc., (5-7).

\section{COMENTARIOS}

A unque en el pasado se hicieron ya algunos ensayos tratando de utilizar corriente bipolar de alta frecuencia para ciertas maniobras endourológicas, éstos no dieron el resultado esperado y fueron desestimados por su inoperancia (8).

$\mathrm{N}$ o ha sido sino hasta el reciente desarrollo de las potentes corrientes de alta frecuencia por pulsos cuyas aplicaciones en cirugía abierta y laparoscópica han constituido un avance considerable al hacer posible el sellado de vasos de grueso calibre y la soldadura de tejidos mediante la fusión del colágeno tisular cuando se ha abierto la posibilidad de mejorar sustancialmente la propia técnica de la resección transuretral, respetando en gran medida sus principios fundamentales.

Cuando tuvimos la ocasión de usar por primera vez en una R.T.U.P. un electrobisturí bipolar por pulsos, nos dimos cuenta de la enorme trascendencia que tendría la incorporación a la práctica diaria de esta nueva tecnología. Sin embargo, las prestaciones de aquel primitivo generador Plasmakinético eran mucho menores que las de los actuales, y las asas, además de pequeñas (tan solo de $3 \mathrm{~mm}$ ), tenían muy poca incurvación.

Este generador una vez perfeccionado proporcionaba un corte con excelente hemostasia por desecación. Sin embargo tenía el defecto de que precisamente por desecar el tejido próximo al corte, cuando se llegaba a tejidos especialmente fibrosos como es la cápsula prostática, su capacidad de corte se veía mermada, resbalando el asa por no formarse adecuadamente el arco de plasma. Los intentos de "apurar" la cápsula en nestas condiciones va asociada a un incremento del número de perforaciones no deseadas. Ello explica el elevado número de perforaciones capsulares que se registraron en el grupo A. Este inconveniente ha quedado totalmente resuelto tras haberse efectuado un reajuste en el programa del nuevo gene- rador "Super Pulsos". N o obstante echamos en falta en este nuevo generador, la existencia de un nuevo botón añadido al pedal, que nos permitiera trabajar durante la primera fase de una R.T.U.P. con el circuito electrónico de su versión anterior (mucho más hemostática).

A pesar de haber utilizado en este estudio la versión Plasmakinética anterior a la "super pulsos", el rendimiento quirúrgico fue significativamente mejor que con la resección convencional. Igualmente, la hemorragia intraoperatoria fue subjetivamente menor en los pacientes del grupo A (al ser un estudio retrospectivo no nos fue posible realizar un cálculo objetivo). $\mathrm{N}$ o obstante, en el grupo A se precisó de la mitad de transfusiones que en grupo $B$.

Al margen de los pacientes integrados en este estudio, hemos tenido también la oportunidad de probar otros equipos bipolares por pulsos aptos para la R.T.U.P.

El equipo Vista ${ }^{\mathrm{TM}}$ de ACMI, tiene una excelente calidad de corte y hemostasia. No obstante encontramos que en determinados momentos (al socavar la celda prostática por ejemplo) el asa más distal dificulta la penetración de la primera. 0 tros inconvenientes que encontramos en este equipo es que el jet de la irrigación no es capaz de dispersar con facilidad las abundantes burbujas generadas con la vaporización tisular, y que el asa más distal se carga de "carbonilla" a lo largo de la resección, lo que obliga a interrumpir ésta para limpiarla.

Las resecciones que hemos hecho con el equipo TURIS TM de Olympus nos permitieron apreciar una magnífica calidad de corte, aunque con la impresión de que le sobra energía al equipo, pues su arco de plasma es demasiado intenso, lo que hace que en ocasiones se rompa el asa durante la resección. Este equipo tiene también una buena capacidad de hemostasia, pero adolece igualmente de cierto defecto en la capacidad de aclarado del medio.

En síntesis podemos decir que la calidad de corte y hemostasia son muy similares con las últimas generaciones de los tres equipos mencionados. Comparando la calidad de corte de los equipos bipolares con los monopolares, podemos afirmar que ésta es muy superior con los primeros, pudiéndose dar cortes extremadamente finos y precisos, con una mínima o nula capacidad de estimulación neuromuscular. Por 
otra parte, la hemostasia, con los equipos bipolares es también superior. Durante la propia resección, la mínima desecación tisular que se produce en las inmediaciones del área de corte (inducida por el plasma) hace que se sellen los vasos más finos, sin el inconveniente que reportaría en un equipo monopolar el empleo de corrientes mixtas (indeseables efectos térmicos en profundidad). La hemostasia con los equipos bipolares se consigue "por desecación tisular". Por ello, al contrario que en una resección convencional, en la bipolar se a precia cómo palidece el tejido con la coagulación, sin que llegue a producirse carbonización ni daño térmico profundo.

La ausencia de inercia térmica hace que no se produzca un cono de tejido desnaturalizado, responsable del molesto cuadro de irritabilidad miccional en el postopera torio inmediato. No obstante, aunque solo nos ha ocurrido en un caso entre cincuenta y un pacientes, la hemorragia tardía de la celda prostática es una complicación que, al menos desde el punto de vista teórico, debería tenerse en cuenta, por ser el sellado vascular muy superficial.

Dadas estas cualidades, con cualquiera de estos equipos bipolares se pueden resecar con gran precisión tumores de próstata 0 de vejiga, consiguiéndose al mismo tiempo que muy buenos fragmentos para estudio histopatológico, una excelente hemostasia y un riesgo caso nulo de provocar perforaciones indeseadas debido a la brusca contracción de los músculos aductores por estímulo eléctrico. Este hecho ha sido comprobado también, aunque de forma experimental por otros autores (9). Aún no hemos tenido la oportunidad de realizar resecciones percutáneas de tumores pielocaliciales con estos nuevos equipos, pero sí que los hemos empleado con éxito en resecciones transuretrales de tumores uroteliales de vejiga y en resecciones trans anales de poliposis rectosigmoideas. En la recientemente pasada época de los "rollerballs" y "rollerbars", la empresa ProSurg diseñó un electrodo bipolar de doble rodillo (Bibar ${ }^{\mathrm{TM}}$ ) con el intento de mejorar la efectividad de la electrovaporización prostática. Esta idea de usar un electrodo doble ha sido aplicada por la empresa ACMI en su actual doble asa para la R.T.U.P bipolar. No obstante, se deben diferenciar muy claramente los conceptos "electrovaporización" del de "electrorresección". Las múltiples de técnicas propuestas en los últimos años para "vaporizar" el tejido prostático, algunas de ellas ejecutadas con la ayuda de un resectoscopio (electrovaporización), pueden inducir a confusión y hacer pensar al lector que la RTUP con el asa PK Plasma - Sect ${ }^{\mathrm{TM}}$ de 4 $\mathrm{mm}$. es una variante más de estas formas de ablación prostática.

La confusión puede ser aún mayor cuando se contrastan opiniones generales referentes al sistema PlasmaKinetc de la firma Gyrus Medical, pues hasta el 11 de mayo del año 2000, la FDA no había aprobado el uso del prototipo inicial de asa de corte (PK Plasma - Sect ${ }^{\mathrm{TM}}$ ), y lo que hasta entonces se había utilizado era el sistema Plasmakinetc con el electrodo tipo rodillo para "vaporizar" la próstata (PK Plasma $V{ }^{\mathrm{T} M}$ ). Las opiniones pues que hasta el momento hayan podido vertirse acerca del sistema Plasmakinetc han hecho siempre referencia al sistema de "vaporización", y no al de "resección". Esto mismo ocurre con otras publicaciones aparecidas recientemente en la literatura $(10,11)$ y en las que la energía electrokinética se utilizó con el rodillo de vaporización (PKVP).

N uestra experiencia con la R.T.U.P. a baja presión hidráulica nos sitúa en una posición ventajosa a la hora de comparar la eficacia de la R.T.U.P. con la de otras alternativas propuestas, incluido el láser de Holmium de alta potencia (que disponemos en nuestro Servicio desde hace 3 años). Hasta ahora nada supera en rendimiento, eficacia y simplicidad a la resección transuretral. Sin embargo, la incorporación de los generadores de corriente bipolar por pulsos mejora las condiciones de seguridad eléctrica de esta cirugía (evitan el riesgo potencial de quemaduras, estenosis uretral, electrocución), reducen las secuelas derivadas del aumento de temperatura en la periferia de la glándula prostática (12) (esclerosis de celda, impotencia, incontinencia urinaria), consiguen el sellado instantáneo de la mayor parte de los vasos prostáticos (disminuyen la hemorragia intraoperatoria) y sobre todo, evitan las complicaciones derivadas de la posible reabsorción de agua o solución de glicina (síndrome de la RTUP, amaurosis).

\section{CONCLUSIONES}

El estudio retrospectivo y comparativo de las resecciones llevadas a cabo con generadores monopolares y bipolares por pulsos nos permite afirmar que estamos ante un verdadero logro tecnológico que 
mejora muy sustancialmente las ventajas de la R.T.U.P., sin mermar en absoluto ninguna de las magníficas cualidades de esta técnica.

A unque aún puede darse un fino ajuste al circuito electrónico de algunos de estos generadores, en el momento presente superan ya con creces las prestaciones de los mejores generadores monopolares.

Con los generadores bipolares por pulsos han pasado a ser un mito barreras de los 50 gramos o la hora de intervención en las R.T.U.P. Si a ello sumamos la posibilidad de poder resecar la próstata a una verdadera baja presión hidráulica (bolsas de irrigación a no más de $40 \mathrm{~cm}$ de altura y camisa de Amplat de 30 Fr. suprapúbica), el riesgo de hiperhidratación del paciente es nulo (con esta técnica no hemos encontrado relación significativa entre el tiempo de resección y la reabsorción de líquido irrigante) . Bajo este prisma, la docencia de la R.T.U.P. se ve muy beneficiada y se puede ampliarse el límite de peso hasta 100 gramos para reseccionistas con cierta experiencia.

\section{BIBUOGRAFIA y IFCTURAS RECOMENDADAS (*lectura de interés y **lectura fundamental)}

*1. CLEMENTE RAMOS, L.M.; RAMASCO RUEDA, F.; PLATAS SANCHO, A. y cols.: "Síndrome de reabsorción post-resección transuretral (R.T.U.) de próstata: revisión de aspectos fisiopatológicos, diagnósticos y terapéuticos". Actas Urol. Esp., 25: 14, 2001.

2. MEBUST, W.K.; HOLTGRAVE, H.L.; COCKETT, A.T.K.: "Transurethral prostatectomy: immediate and postoperative complications. A cooperative study of 13 participating institutions evaluating 3855 patients". J. Urol., 141: 243, 1989.
**3. VALDIVIA URÍA, J.G.; SÁNCHEZ ZALABARDO, M.; REGOJO ZAPATA, O. y cols.: "La resección transuretral de la próstata mediante corriente bipolar por pulsos”. En Leiva O, Angulo J, González J, editores. Hipertrofia Prostática Benigna. Madrid: Luzán 5, S A. p. 507, 2003.

4. MILES, J.B.; SIRLS, L.: "Transurethral electrovaporization of the prostate". En Kirby R, Mc Connell JD, Fitzpatrick JM, Roehrborn CG, Boyle P, editores. Texbook of benign prostatic hyperplasia. Oxford: Isis Medical Media Ltd. p. 463, 1996.

5. NARAYAN, P.; FOURMIER, G.; INDUDHARA, R. y cols.: "Transuretral evaporation of prostate with $\mathrm{Nd}$ :YAG laser using contact free beam technique: Results in 61 patients with benign prostatic hyperplasia". Urology, 43: 813, 1994.

6. COSTEllO, A.J.; BOWSHER, W.G.; BOLTON, D.M. y cols.: "Laser ablation of the prostate in patients with benign prostatic hypertrophy". J. Urol., 69: 603, 1992.

**7. KÜPELI, S.; YILMAZ, E.; SOYGÜR, T. y cols.: "Randomized study of transuretral resection of the prostate and combined transuretral resection and vaporization of the prostate as a therapeutic alternative in men with benign prostatic hyperplasia”. J. Endourol., 15: 317, 2001.

8. VICENTE, J.: "Corriente bipolar en cirugía endoscópica urológica". Actas II Congress of Endoscopic Urologic Surgery. Bristol,. p. 17, 1981.

*9. SHIOZAWA, H.; AIZAWA, T.; ITU, T. y cols.: "A new transurethral resection system: operating in saline environment precludes obturator nerve reflexes". J. Urol., 168: 2665, 2002.

*10. BOTTO, H.; LEBRET, T.; MARRÉ, P.H. y cols.: "Electrovaporization of the prostate with the Gyrus device". J. Endourol., 15: 313, 2001.

*11. EATON, A.C.; FRANCIS, R.N.: "The provisión of transuretral prostatectomy on a day-case basis using bipolar plasma kinetic technology". BJU Int., 89: 534, 2002.

12. VICENTE, J.: "Cirugía endoscópica de la próstata". En Vicente J, editor: Tratado de Endurología. Barcelona: Pulso ediciones S A. p. 303, 1996. 